\title{
Spatial and temporal dimensions of butterfly species diversity in Jahangirnagar University campus and its suburbs, Bangladesh
}

\author{
Farjana Akter, Mirza Shamim Ahasan Habib and Md. Monwar Hossain* \\ Department of Zoology, Jahangirnagar University, Savar, Dhaka, Bangladesh
}

\begin{abstract}
Survey was conducted in four different areas viz. Jahanginagar University Campus, Gerua, Nabinagar and Chandra of Bangladesh from October 2012 to September 2013. A total of 100 species of butterflies under 9 families were identified. The maximum number of butterflies (31 species) was recorded under the family Lycaenidae followed by Nymphalidae (17 species), Pieridae (16 species), Hesperiidae (15 species), Satyridae (7 species), Papilionidae ( 7 species), Danaidae (5 species) and 1 species to each family of Acraediae and Amathusiidae. Species diversity was higher from summer to early monsoon and declined in late winter. Out of four areas, the highest number of butterfly was recorded at Jahangirnagar University campus followed by Gerua, Nabinagar and the lowest at Chandra. Out of 100 species, 7 were very common, 12 were common, 17 were rare, 64 were very rare. Dusky Part Wing (Psolos fuligo Mabille, 1876) under the family Hesperiidae was recorded for the first time in Bangladesh.
\end{abstract}

Key words: Spatial and temporal dimensions, butterflies, JU campus, suburbs

\section{INTRODUCTION}

Butterfly is one of the most wonderful and fascinating smaller creatures on earth. It has been increasingly recognized that butterflies are important for ecological and conservation monitoring because of its strong sensitivity to any changes of climatic conditions as well as seasonal and ecological changes (Kunte 1997; Houlahan et al., 2000; Mac Nally et al., 2004; Stuart et al., 2004). Adult butterflies function as vital plant pollinators in the environment causing pollination to more than 50 economically important plant crops (Borges et al., 2003, Rajagopal et al., 2011). However, it is a matter of regret that they are not taken into account under conservation efforts at broad way.

It is estimated that approximately 28,000 species of butterflies are found to inhabit in the world (Robbins \& Opler, 1997) while it is assumed that there are about 400 species of butterflies exists in Bangladesh (Larsan, 2004; Chowdhury \& Hossain, 2013). Of which, 325 species have been identified in Bangladesh (Ameen \& Chowdhury, 1968; Baksha \& Choudhury, 1983; Baksha \& Choudhury, 1985; Alam \& Ullah, 1995; Chowdhury \& Mohiuddin, 2003; Hossain et al., 2003; Larsan , 2004; Bashar et al., 2006; Razzak et al., 2007; Ahmad et al., 2009; Shefa \& Hossain, 2010; Islam et al., 2011; Habib et al., 2012; Habib et al., 2013; Chowdhury \& Hossain, 2013; Islam et al., 2013; Khandokar et al., 2013; Neogi et al., 2014; Bashar, 2014; Hossain, 2014; Hossain, 2014a, Hossain, 2014b). On the other hand, about 110 species of butterflies were recorded in Jahangirnagar University campus and many more remain to be identified in this area (Hossain et al.,

\footnotetext{
* Corresponding author. E-mail: zona444@yahoo.com
} 
2003; Razzak et al., 2007; Shefa \& Hossain, 2010; Chowdhury \& Hossain, 2013). Thus, present study was started to evaluate the species diversity, dynamics, ecological status of within and around Jahangirnagar University campus.

\section{MATERIALS AND METHOD}

Study sites: The study areas are geographically situated in central part of Bangladesh under Dhaka and Gazipur district. The study sites were selected depending on their vegetation characteristics and human interferences. The temperature was highest in April $\left(36.3^{\circ} \mathrm{C}\right)$ and lowest in January $\left(7.7^{\circ} \mathrm{C}\right)$. The total rainfall during the study period was $1217 \mathrm{~mm}$ and the mean monthly humidity was $82.07 \%$. Description of the study sites are given below:

Jahangirnagar University campus (J): The campus of Jahangirnagar University is an undeclared sanctuary. Geographically the campus is at $23^{\circ} 5243.89^{\prime} \mathrm{N}$ latitude and $90^{\circ} 1617.10^{\prime}$ E longitude, $32 \mathrm{~km}$ north-west from Dhaka City which includes about 280 hectors of area and it is about 6 meter high from mean sea level (Fig. 1). There are many woodlands, grasslands, garden, vegetable garden, rice field and bushes which are the habitat of many insects and wildlife including butterflies. All of these plants are very important for butterfly as larval host plant and nectar plants.

Gerua (G): It $\left(23^{\circ} 5202.61^{\prime} \mathrm{N}\right.$ latitude and $90^{\circ} 1247.70^{\prime} \mathrm{E}$ longitude) is adjacent to Jahangirnagar University campus (Fig: 1). It is also enriched with reddish and yellowish soil with large amount of iron, aluminum and calcium. From the month of June to August, east side of gerua bazaar (crop field site) covered with rainwater. Gerua consists of some cultivated lands, homestead vegetation and bushes.

Nabinagar (National Martyr's Monument and Kurgao area) (N): Geographically it $\left(23^{\circ} 5443.87^{\prime} \mathrm{N}\right.$ latitude and $90^{\circ} 1513.05^{\prime} \mathrm{E}$ longitude) is closed to Jahangirnagar University campus (Fig. 1). It is enriched with red soil. Nabinagar is most human produced disturbed area. This area is bounded by Savar Cantonment Golf course on south and Jahangirnagar University campus on south east, Chandra-Nabinagr road on north- east, Ganakbari on north, Gono Shastha and Bangshi river on west.

Chandra (Sal Forest Area) (C): Chandra, the moist deciduous Sal (Shorea robusta) forest is distributed at the Kaliakur Upazila in Gazipur district. Geographically it $\left(24^{\circ} 0300.66^{\prime} \mathrm{N}\right.$ latitude and $90^{\circ} 1414.93^{\prime} \mathrm{E}$ longitude) is closed to Nabinagar area (Fig.1). It is characterized by high, undulated land surface with red soil. Sal plants were more common in this area, but at present these natural trees are replaced by exotic plant species (Acacia sp.). Chandra area is divided into eastern and western part by Nabinagar Chandra road. BKSP (Bangladesh Krira Shikkha Proothistan), Nandan Park and EPZ (export processing zone) situated on south of this area.

Sampling methods: Butterflies were collected from morning 8:30 am to evening 5:00 pm during October 2012 to September 2013. However, counting days were changed during unfavorable weather condition. Similar transect-line was set up at each site. 
Collection: Butterflies were collected using a hand-held sweeping net and placed in killing jar. Chloroform was used as killing agent. After killing, they were taken into triangular paper envelope to give a perfect using shape. At last, specimens were taken in laboratory at Department of Zoology, Jahangirnagar University for further confirmation of identification and preservation.

Identification: The identification of butterflies was conducted by using the keys of Bingham (1905), Bingham (1907), Evans (1932), Wynter-Blyth (1957) and Talbot (1978 $\mathrm{a}, \mathrm{b})$.

For convenience of data collection and interpretation, the year was divided into six seasons as, (a) early winter (October and November), (b) late winter (December and January), (c) spring (February and March), (d) summer (April and May), (e) early monsoon (June and July) and (f) late monsoon (August and September). The occurrence of butterfly indicated by 1-12 number (in Table 1) depending on the different study period (month) that comprises by October (10), November (11), December (12), January (1), February (2), March (3), April (4), May (5), June (6), July (7), August (8) and September (9).

For assessing butterfly status, the observed butterflies species were grouped into four categories to indicate the status of assessment namely : Very common (VC) : presence of 35 to 50 butterfly per month, Common (C) : presence of 20 to 35 butterfly per month , Rare (R) : presence of 10 to 20 butterfly per month and Very Rare (VR) : presence of 1 to 10 butterfly per month.

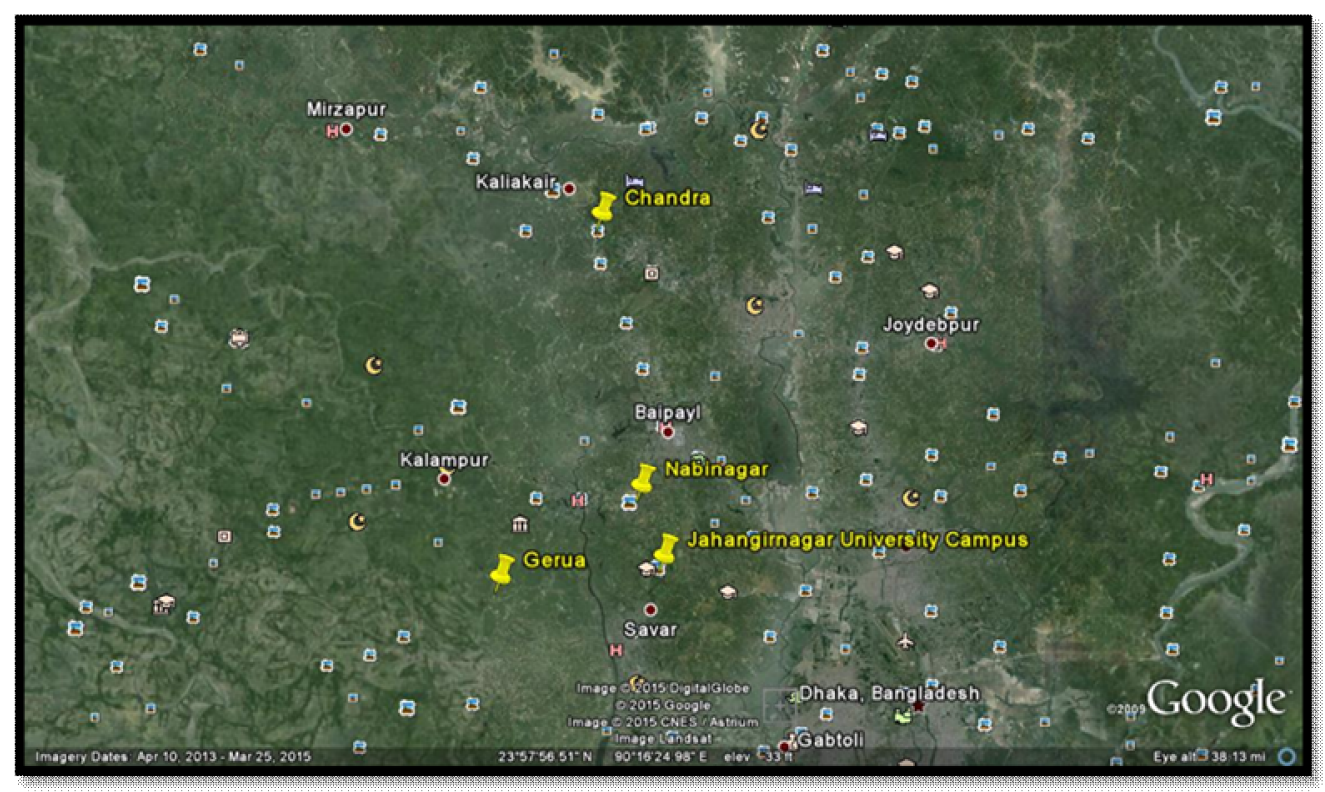

Fig. 1. Map of four different study areas (Yellow marks) 


\section{RESULTS AND DISCUSSION}

During the period of study, a total of 100 species of butterflies under 9 families were recorded. Among them, 17 species belonged to the family Nymphalidae, 16 species to Pieridae, 31 Species to Lycaenidae, 7 species to Satyridae, 7 species to Papilionidae, 5 species to Danaidae, 15 species to Hesperiidae and 1 species to each family of Acraediae and Amathusidae in JU campus, Gerua, Nabinagar and Chandra (Table 1, Fig. 2). Out of 100 identified species, 1 species, Dusky Part Wing (Psolos fuligo Mabille, 1876) under the family Hesperiidae was newly recorded species in Bangladesh (Plate 1). This species found at bushes and preferred shady areas. Besides Bangladesh, this species is also found in India, Laos, Malaysia, Philippines, Singapore, Thailand and Vietnam (Choudhury \& Hossain, 2013).

In JU campus a total of 98 species (except Rapala iarbus and Arhopala amantes) under 9 families were recorded (Table 1). On the other hand, 71 species of butterflies under 8 families, 69 species under 8 families and 68 species under 8 families were recorded from Gerua, Nabinagar and Chandra respectively, (Table 1 and 2).

In this investigation, out of 100 species, 7 species were very common (Junonia atlites, Junonia almana, Eurema hecabe, Zizina otis sangara, Melanitis leda, Mycaelesis perseus blasius, Parnara guttata), 12 species were common (Phalanta phalantha, Neptis hylas, Junonia lemonias, Leptosia nina, Catopsilia Pomona, Castalius rosimon rosimon, Prosotas dubiosa, Rapala manea schitacea, Neopithecops zalmora, Elymnias hypermnestra, Papilio polytes, Iambrix salsala), 17 species were rare and 64 species were very rare (Table 1$)$.

Out of 100 species, 27 were found throughout the year in all study areas and the species were Junonia atlites, Junonia almana, Phalanta phalantha, Cethosia cyane, Neptis hylas, Leptosia nina, Eurema hecabe, Delias eucharis, Castalius rosimon rosimon, Prosotas dubiosa, Rapala manea, Chilades laius laius, Zizula hylax, Remelana jangala, Zizina otis sangara, Zizzeria maha, Melanitis leda, Mycaelesis perseus blasius, Mycaelesis perseus, Elymnias hypermnestra, Mycaelesis mineus, Graphium agamemnon, Papilio polytes, Graphium doson, Papilio demolius, Parnara guttata, Telicota ancilla bambusae and the rest of the other species were season specific (Table 1). Butterflies did not occur evenly throughout the year and species abundance was gradually increased in the month of November, 2012. On the other hand the highest species diversity documented during March to April, 2013 whereas the lowest species diversity in January, 2013 (Table 3).

Table 1. Status, abundance, occurrence and distribution of butterfly in JU campus and its suburbs

\begin{tabular}{l|l|l|c|c|c|c}
\hline $\begin{array}{l}\text { Sl. } \\
\text { no }\end{array}$ & Common name & Scientific name & Status & $\begin{array}{c}\text { Abundance } \\
(\text { average })\end{array}$ & $\begin{array}{c}\text { Occurrence } \\
(\text { months })\end{array}$ & Distributio \\
\hline \multicolumn{7}{l}{ Nymphalidae } \\
\hline 1 & Yellow Pansy & Junonia hierta Fab. & VR & 7.9 & $2-5$ & J,G,N \\
2 & Grey Pansy & Junonia atlites Linn. & VC & 38.8 & $10-9$ & J,G,N,C
\end{tabular}




\begin{tabular}{|c|c|c|c|c|c|c|}
\hline 3 & Peacock Pansy & Junonia almana Linn. & $\mathrm{VC}$ & 39.4 & $10-9$ & $\mathrm{~J}, \mathrm{G}, \mathrm{N}, \mathrm{C}$ \\
\hline 4 & Common Leopard & $\begin{array}{l}\text { Phalanta phalantha } \\
\text { Drury }\end{array}$ & $\mathrm{C}$ & 23.6 & $10-9$ & $\mathrm{~J}, \mathrm{G}, \mathrm{N}, \mathrm{C}$ \\
\hline 5 & The Commander & $\begin{array}{l}\text { Moduza procris } \\
\text { Cramer }\end{array}$ & VR & 1 & $11-12,5,8$ & $\mathrm{~J}, \mathrm{G}$ \\
\hline 6 & Leopard Lacewing & Cethosia cyane Drury & $\mathrm{R}$ & 11.6 & $10-9$ & $\mathrm{~J}, \mathrm{G}, \mathrm{N}, \mathrm{C}$ \\
\hline 7 & Common Castor & $\begin{array}{l}\text { Ariadne merione } \\
\text { Moore }\end{array}$ & VR & 6 & $10,2-9$ & $\mathrm{~J}, \mathrm{G}, \mathrm{N}, \mathrm{C}$ \\
\hline 8 & Angled Castor & Ariadne ariadne Linn. & VR & 5.3 & $10,2-5,7-9$ & $\mathrm{~J}, \mathrm{G}, \mathrm{N}, \mathrm{C}$ \\
\hline 9 & Common Sailor & Neptis hylas Linn. & $\mathrm{C}$ & 21 & $10-9$ & $\mathrm{~J}, \mathrm{G}, \mathrm{N}, \mathrm{C}$ \\
\hline 10 & $\begin{array}{l}\text { Chestnut Streaked } \\
\text { Sailor }\end{array}$ & Neptis jumbah Moore & VR & 2.7 & $11-12,2-7$ & $\mathrm{~J}$ \\
\hline 11 & Common Sergeant & Athyma opalina Linn. & VR & 3.5 & $\begin{array}{l}10-12,2-5 \\
7\end{array}$ & $\mathrm{~J}, \mathrm{G}, \mathrm{N}, \mathrm{C}$ \\
\hline 12 & Lemon Pansy & $\begin{array}{l}\text { Junonia lemonias } \\
\text { Linn. }\end{array}$ & $\mathrm{C}$ & 20.5 & $10,2-9$ & $\mathrm{~J}, \mathrm{G}, \mathrm{N}, \mathrm{C}$ \\
\hline 13 & Great Eggfly & Hypolimnas bolina Linn. & VR & 6.8 & $10-12,2-9$ & $\mathrm{~J}, \mathrm{G}, \mathrm{N}, \mathrm{C}$ \\
\hline 14 & Common Baron & $\begin{array}{l}\text { Euthalia aconthea } \\
\text { Moore }\end{array}$ & VR & 4.9 & $10-11,2-9$ & $\mathrm{~J}, \mathrm{G}, \mathrm{N}, \mathrm{C}$ \\
\hline 15 & Powdered Baron & $\begin{array}{l}\text { Euthalia monina } \\
\text { Moore }\end{array}$ & VR & 0.1 & 4 & $\mathrm{~J}$ \\
\hline 16 & Gaudy Baron & $\begin{array}{l}\text { Euthalia lubentina } \\
\text { Fruhstorfer }\end{array}$ & VR & 0.1 & 4 & $\mathrm{~J}$ \\
\hline \multirow[t]{2}{*}{17} & Blue Pansy & Junonia orithya Linn. & VR & 0.2 & 3,5 & $\mathrm{~J}$ \\
\hline & Pieridae & & & & & \\
\hline 18 & Psyche & Leptosia nina Fab. & $\mathrm{C}$ & 28.8 & $10-9$ & $\mathrm{~J}, \mathrm{G}, \mathrm{N}, \mathrm{C}$ \\
\hline 19 & Striped Albatross & $\begin{array}{l}\text { Appias libythea } \\
\text { Swinhoe }\end{array}$ & $\mathrm{R}$ & 15.8 & $10-12,2-9$ & $\mathrm{~J}, \mathrm{G}, \mathrm{N}, \mathrm{C}$ \\
\hline 20 & Common Emigrant & Catopsilia pomona Fab. & $\mathrm{C}$ & 25.6 & $10-12,2-9$ & $\mathrm{~J}, \mathrm{G}, \mathrm{N}, \mathrm{C}$ \\
\hline 21 & Mottled Emigrant & $\begin{array}{l}\text { Catopsilia pyranthe } \\
\text { Linn. }\end{array}$ & VR & 9.9 & $10-12,2-9$ & $\mathrm{~J}, \mathrm{G}, \mathrm{N}, \mathrm{C}$ \\
\hline 22 & Common Gull & $\begin{array}{l}\text { Cepora nerissa nerissa } \\
\text { Fab. }\end{array}$ & $\mathrm{R}$ & 15.7 & $10-11,2-9$ & $\mathrm{~J}, \mathrm{G}, \mathrm{N}, \mathrm{C}$ \\
\hline 23 & $\begin{array}{l}\text { Common Grass } \\
\text { Yellow }\end{array}$ & Eurema hecabe Linn. & $\mathrm{VC}$ & 49.1 & $10-9$ & $\mathrm{~J}, \mathrm{G}, \mathrm{N}, \mathrm{C}$ \\
\hline 24 & Common Jezebel & Delias eucharis Drury & $\mathrm{R}$ & 12.6 & $10-9$ & $\mathrm{~J}, \mathrm{G}, \mathrm{N}, \mathrm{C}$ \\
\hline 25 & Red Spot Jezebel & $\begin{array}{l}\text { Delias descombesi } \\
\text { Boisduval }\end{array}$ & VR & 0.5 & $3-4$ & $\mathbf{J}$ \\
\hline 26 & Common Wanderer & $\begin{array}{l}\text { Pareronia valeria } \\
\text { Cramer }\end{array}$ & VR & 6.1 & $\begin{array}{l}11-12,2-6 \\
8\end{array}$ & $\mathrm{~J}, \mathrm{G}, \mathrm{N}, \mathrm{C}$ \\
\hline 27 & Pale Wanderer & $\begin{array}{l}\text { Pareronia anais } \\
\text { Lesson }\end{array}$ & VR & 8.1 & $10-12,2-7$ & $\mathrm{~J}, \mathrm{G}, \mathrm{N}, \mathrm{C}$ \\
\hline 28 & Chocolate Albatross & Appias lyncida Cramer & VR & 3.6 & $2-6,9$ & $\mathrm{~J}, \mathrm{G}, \mathrm{N}, \mathrm{C}$ \\
\hline 29 & Painted Jezebel & Delias hyparete Linn & VR & 1.8 & $2-4$ & $\mathrm{~J}, \mathrm{G}, \mathrm{N}, \mathrm{C}$ \\
\hline 30 & Great Orange Tip & $\begin{array}{l}\text { Hebomoia glaucippe } \\
\text { Linn. }\end{array}$ & VR & 0.8 & $11-12,2-4$ & $\mathrm{~J}$ \\
\hline 31 & $\begin{array}{l}\text { Indian Cabbage } \\
\text { White }\end{array}$ & $\begin{array}{l}\text { Arlogeia canidia } \\
\text { Evans }\end{array}$ & VR & 1.3 & $12-1$ & G \\
\hline 32 & Lemon Emigrant & $\begin{array}{l}\text { Catopsilia crocale } \\
\text { Cramer }\end{array}$ & VR & 5.4 & $3-5,9$ & $\mathrm{~J}$ \\
\hline
\end{tabular}




\begin{tabular}{|c|c|c|c|c|c|c|}
\hline 33 & $\begin{array}{l}\text { Three Spot Grass } \\
\text { Yellow }\end{array}$ & $\begin{array}{l}\text { Eurema blanda } \\
\text { Wallace }\end{array}$ & VR & 2 & $3-4$ & $\mathrm{~J}$ \\
\hline & \multicolumn{6}{|l|}{ Lycaenidae } \\
\hline 34 & Common Pierrot & Castalius rosimon Fab. & $\mathrm{C}$ & 33.9 & $10-9$ & $\mathrm{~J}, \mathrm{G}, \mathrm{N}, \mathrm{C}$ \\
\hline 35 & Stripped Pierrot & Tarucus nara Kollar & VR & 5.5 & $10-12,2-9$ & $\mathrm{~J}, \mathrm{G}, \mathrm{N}, \mathrm{C}$ \\
\hline 36 & Spotted Pierrot & $\begin{array}{l}\text { Tarucus callinara } \\
\text { Butler }\end{array}$ & VR & 2.3 & $10-12,2-7$ & $\mathrm{~J}, \mathrm{G}, \mathrm{N}$ \\
\hline 37 & Angled Pierrot & $\begin{array}{l}\text { Caleta caleta } \\
\text { Hewitson }\end{array}$ & $\mathrm{R}$ & 11 & $10,2-9$ & $\mathrm{~J}, \mathrm{G}, \mathrm{N}, \mathrm{C}$ \\
\hline 38 & Common Line Blue & $\begin{array}{l}\text { Prosotas nora ardates } \\
\text { Moore }\end{array}$ & VR & 8.4 & $10-12,2-9$ & $\mathrm{~J}, \mathrm{G}, \mathrm{N}, \mathrm{C}$ \\
\hline 39 & Tailless Line Blue & $\begin{array}{l}\text { Prosotas dubiosa } \\
\text { Evans }\end{array}$ & $\mathrm{C}$ & 21.2 & $10-9$ & $\mathrm{~J}, \mathrm{G}, \mathrm{N}, \mathrm{C}$ \\
\hline 40 & Dark Grass Blue & $\begin{array}{l}\text { Zizerria karsandra } \\
\text { Moore }\end{array}$ & VR & 6.2 & $2-9$ & $\mathrm{~J}, \mathrm{G}, \mathrm{N}, \mathrm{C}$ \\
\hline 41 & Slate Flash & Rapala manea Moore & $\mathrm{C}$ & 25.9 & $10-9$ & $\mathrm{~J}, \mathrm{G}, \mathrm{N}, \mathrm{C}$ \\
\hline 42 & Pea Blue & $\begin{array}{l}\text { Lampides boeticus } \\
\text { Linn. }\end{array}$ & VR & 2.1 & $10,2-6$ & $\mathrm{~J}, \mathrm{G}$ \\
\hline 43 & Lime Blue & $\begin{array}{l}\text { Chilades laius laius } \\
\text { Cramer }\end{array}$ & VR & 9.1 & $10-9$ & $\mathrm{~J}, \mathrm{G}, \mathrm{N}, \mathrm{C}$ \\
\hline 44 & The Quaker & $\begin{array}{l}\text { Neopithecops zalmora } \\
\text { Butler }\end{array}$ & $\mathrm{C}$ & 20.7 & $10,12-9$ & $\mathrm{~J}, \mathrm{G}, \mathrm{N}, \mathrm{C}$ \\
\hline 45 & Forget me not & $\begin{array}{l}\text { Catochrysops strabo } \\
\text { Fab. }\end{array}$ & VR & 1.8 & $10-11,2-5$ & $\mathrm{~J}, \mathrm{G}$ \\
\hline 46 & Tiny Grass Blue & Zizula hylax Fab. & $\mathrm{R}$ & 15.4 & $10-9$ & $\mathrm{~J}, \mathrm{G}, \mathrm{N}, \mathrm{C}$ \\
\hline 47 & Common Silverline & Spindasis vulcanus Fab. & VR & 4 & $\begin{array}{l}10-11,2,4- \\
9\end{array}$ & $\mathrm{~J}, \mathrm{G}, \mathrm{N}$ \\
\hline 48 & Shot Silverline & $\begin{array}{l}\text { Spindasis ictis } \\
\text { Hewitson }\end{array}$ & VR & 2.7 & $10,2-6$ & $\mathrm{~J}, \mathrm{~N}, \mathrm{C}$ \\
\hline 49 & $\begin{array}{l}\text { Long Banded } \\
\text { Silverline }\end{array}$ & $\begin{array}{l}\text { Spindasis lohita } \\
\text { Horsefield }\end{array}$ & VR & 0.2 & $10-11$ & $\mathbf{J}$ \\
\hline 50 & Indian Cupid & $\begin{array}{l}\text { Everes lacturnus } \\
\text { Godart }\end{array}$ & VR & 5.5 & $10,3-8$ & $\mathrm{~J}, \mathrm{G}, \mathrm{N}, \mathrm{C}$ \\
\hline 51 & Common Cerulean & Jamides celeno Cramer & VR & 1.9 & $11,2,4,6-7$ & $\mathrm{~J}, \mathrm{~N}, \mathrm{C}$ \\
\hline 52 & Yamfly & $\begin{array}{l}\text { Loxura atymnus } \\
\text { Fruhstorfer }\end{array}$ & VR & 1.3 & $12,2,6-9$ & $\mathrm{~J}, \mathrm{G}$ \\
\hline 53 & Chocolate Royal & $\begin{array}{l}\text { Remelana jangala } \\
\text { Moore }\end{array}$ & VR & 5.2 & $10-9$ & $\mathrm{~J}, \mathrm{G}, \mathrm{N}, \mathrm{C}$ \\
\hline 54 & Monkey Puzzle & Rathinda amor Fab. & VR & 2.1 & $11,2-3,6-9$ & $\mathrm{~J}$ \\
\hline 55 & Lesser Grass Blue & $\begin{array}{l}\text { Zizina otis sangara } \\
\text { Moore }\end{array}$ & $\mathrm{VC}$ & 40.3 & $10-9$ & $\mathrm{~J}, \mathrm{G}, \mathrm{N}, \mathrm{C}$ \\
\hline 56 & Pale Grass Blue & Zizzeria maha Kollar & $\mathrm{R}$ & 16.8 & $10-9$ & $\mathrm{~J}, \mathrm{G}, \mathrm{N}, \mathrm{C}$ \\
\hline 57 & Centaur Oakblue & $\begin{array}{l}\text { Arhopala centaurus } \\
\text { Moore }\end{array}$ & VR & 0.2 & 5 & $\mathrm{~J}$ \\
\hline 58 & Large Oakblue & $\begin{array}{l}\text { Arhopala amantes } \\
\text { Hewitson }\end{array}$ & VR & 0.1 & 12 & $\mathrm{C}$ \\
\hline 59 & Ape fly & $\begin{array}{l}\text { Spalgis epius epius } \\
\text { Westwood }\end{array}$ & VR & 0.5 & $5-6,9$ & $\mathrm{~J}$ \\
\hline 60 & $\begin{array}{l}\text { Common Red } \\
\text { Flash }\end{array}$ & Rapala iarbus Fab. & VR & 0.1 & 11 & $\mathrm{C}$ \\
\hline
\end{tabular}




\begin{tabular}{|c|c|c|c|c|c|c|}
\hline 61 & $\begin{array}{l}\text { Common Acacia } \\
\text { Blue }\end{array}$ & $\begin{array}{l}\text { Surendra quercetorum } \\
\text { Moore }\end{array}$ & VR & 0.7 & $2-3,9$ & $\mathrm{~J}$ \\
\hline 62 & Pointed Ciliate Blue & $\begin{array}{l}\text { Anthene lycaenina } \\
\text { Felder }\end{array}$ & VR & 1 & $11-12$ & $\mathrm{~J}$ \\
\hline 63 & Metallic Cerulean & Jamides alecto Felder & VR & 0.3 & 10,6 & $\mathrm{~J}$ \\
\hline \multirow[t]{2}{*}{64} & Gram Blue & $\begin{array}{l}\text { Euchrysops cnejus } \\
\text { Fab. }\end{array}$ & VR & 0.2 & 7 & $\mathrm{C}$ \\
\hline & Satyridae & & & & & \\
\hline 65 & $\begin{array}{l}\text { Common Evening } \\
\text { Brown }\end{array}$ & Melanitis leda Linn. & $\mathrm{VC}$ & 52.5 & $10-9$ & $\mathrm{~J}, \mathrm{G}, \mathrm{N}, \mathrm{C}$ \\
\hline 66 & $\begin{array}{l}\text { Common } \\
\text { Bushbrown }\end{array}$ & $\begin{array}{l}\text { Mycaelesis perseus } \\
\text { blasius } \text { Fab. }\end{array}$ & $\mathrm{VC}$ & 37.3 & $10-9$ & $\mathrm{~J}, \mathrm{G}, \mathrm{N}, \mathrm{C}$ \\
\hline 67 & Dingy Bushbrown & $\begin{array}{l}\text { Mycaelesis perseus } \\
\text { Linn. }\end{array}$ & $\mathrm{R}$ & 19.8 & $10-9$ & $\mathrm{~J}, \mathrm{G}, \mathrm{N}, \mathrm{C}$ \\
\hline 68 & Common Palmfly & $\begin{array}{l}\text { Elymnias } \\
\text { hypermnestra Linn. }\end{array}$ & $\mathrm{C}$ & 20.8 & $10-9$ & $\mathrm{~J}, \mathrm{G}, \mathrm{N}, \mathrm{C}$ \\
\hline 69 & $\begin{array}{l}\text { Dark Brand } \\
\text { Bushbrown }\end{array}$ & $\begin{array}{l}\text { Mycaelesis mineus } \\
\text { Linn. }\end{array}$ & VR & 7.1 & $10-9$ & $\mathrm{~J}, \mathrm{G}, \mathrm{N}, \mathrm{C}$ \\
\hline 70 & Lepcha Bushbrown & $\begin{array}{l}\text { Mycaelesis lepcha } \\
\text { Moore }\end{array}$ & VR & 8.1 & $\begin{array}{l}11-12,2-3, \\
5-9\end{array}$ & $\mathrm{~J}, \mathrm{G}, \mathrm{N}, \mathrm{C}$ \\
\hline \multirow[t]{2}{*}{71} & Bamboo Treebrown & $\begin{array}{l}\text { Lethe europa } \\
\text { Fruhstorfer }\end{array}$ & VR & 6.6 & $10-6$ & $\mathrm{~J}, \mathrm{G}, \mathrm{N}, \mathrm{C}$ \\
\hline & Papilionidae & & & & & \\
\hline 72 & Tailed Jay & $\begin{array}{l}\text { Graphium agamemnon } \\
\text { Linn. }\end{array}$ & VR & 9.8 & $10-9$ & $\mathrm{~J}, \mathrm{G}, \mathrm{N}, \mathrm{C}$ \\
\hline 73 & Common Mormon & Papilio polytes Linn. & $\mathrm{C}$ & 22.7 & $10-9$ & $\mathrm{~J}, \mathrm{G}, \mathrm{N}, \mathrm{C}$ \\
\hline 74 & Common Rose & $\begin{array}{l}\text { Pachliopta } \\
\text { aristolochiae Fab. }\end{array}$ & VR & 5.7 & $10-12,2-6$ & $\mathrm{~J}, \mathrm{G}, \mathrm{N}, \mathrm{C}$ \\
\hline 75 & Blue Mormon & $\begin{array}{l}\text { Papilio polymnestor } \\
\text { Cramer }\end{array}$ & $\mathrm{R}$ & 10.7 & $11-12,3-9$ & $\mathrm{~J}, \mathrm{G}, \mathrm{N}, \mathrm{C}$ \\
\hline 76 & Common Jay & $\begin{array}{l}\text { Graphium doson } \\
\text { Felder }\end{array}$ & $\mathrm{R}$ & 14.3 & $10-9$ & $\mathrm{~J}, \mathrm{G}, \mathrm{N}, \mathrm{C}$ \\
\hline 77 & Common Mime & Chilasa clytia Linn. & VR & 9.4 & $10-12,2-9$ & $\mathrm{~J}, \mathrm{G}, \mathrm{N}, \mathrm{C}$ \\
\hline \multirow[t]{2}{*}{78} & Lime Swallow tail & Papilio demolius Linn. & $\mathrm{R}$ & 19.8 & $10-9$ & $\mathrm{~J}, \mathrm{G}, \mathrm{N}, \mathrm{C}$ \\
\hline & Danaidae & & & & & \\
\hline 79 & Blue Tiger & $\begin{array}{l}\text { Tirumala limniace } \\
\text { Cramer }\end{array}$ & VR & 8.3 & $10-12,2-9$ & $\mathrm{~J}, \mathrm{G}, \mathrm{N}, \mathrm{C}$ \\
\hline 80 & Plain Tiger & $\begin{array}{l}\text { Danaus chrysippus } \\
\text { Linn. }\end{array}$ & $\mathrm{R}$ & 11.9 & $11-1,3-9$ & $\mathrm{~J}, \mathrm{G}, \mathrm{N}, \mathrm{C}$ \\
\hline 81 & Striped Tiger & $\begin{array}{l}\text { Danaus genutia } \\
\text { Cramer }\end{array}$ & VR & 7.9 & $10-12,2-9$ & $\mathrm{~J}, \mathrm{G}, \mathrm{N}, \mathrm{C}$ \\
\hline 82 & Common Crow & Euploea core Cramer & $\mathrm{R}$ & 12.3 & $10-12,3-8$ & $\mathrm{~J}, \mathrm{G}, \mathrm{N}, \mathrm{C}$ \\
\hline \multirow[t]{2}{*}{83} & Glassy Tiger & $\begin{array}{l}\text { Danaus genutia aglia } \\
\text { Cramer }\end{array}$ & VR & 3.5 & $11-12,3-7$ & $\mathrm{~J}, \mathrm{C}$ \\
\hline & Hesperiidae & & & & & \\
\hline 84 & Common Snow Flat & $\begin{array}{l}\text { Tagiades japetus } \\
\text { Moore }\end{array}$ & VR & 8.4 & $10-12,2-9$ & $\mathrm{~J}, \mathrm{G}, \mathrm{N}, \mathrm{C}$ \\
\hline 85 & Straight Swift & $\begin{array}{l}\text { Parnara guttata } \\
\text { Snellen }\end{array}$ & $\mathrm{VC}$ & 41.8 & $10-9$ & $\mathrm{~J}, \mathrm{G}, \mathrm{N}, \mathrm{C}$ \\
\hline
\end{tabular}




\begin{tabular}{|c|c|c|c|c|c|c|}
\hline 86 & Chestnut Bob & Iambrix salsala Moore & $\mathrm{C}$ & 26.9 & $10-12,2-8$ & $\mathrm{~J}, \mathrm{G}, \mathrm{N}, \mathrm{C}$ \\
\hline 87 & Grass Demon & Udaspes folus Cramer & VR & 0.5 & $2-3,6$ & $\mathrm{~J}, \mathrm{~N}$ \\
\hline 88 & Chestnut Angle & $\begin{array}{l}\text { Odontoptilum } \\
\text { angulata ,Felder }\end{array}$ & VR & 0.2 & $2-3$ & $\mathrm{~J}$ \\
\hline 89 & Brown Awl & $\begin{array}{l}\text { Badamia } \\
\text { exclamationis Fab. }\end{array}$ & $\mathrm{R}$ & 16.3 & $2-8$ & $\mathrm{~J}, \mathrm{G}, \mathrm{N}$ \\
\hline 90 & Common Red Eye & Matapa aria Moore & VR & 3.5 & $11,2-4,6-9$ & $\mathrm{~J}, \mathrm{G}, \mathrm{N}, \mathrm{C}$ \\
\hline 91 & Contiguous Swift & $\begin{array}{l}\text { Polytremis lubricans } \\
\text { Herrich-Schaffer }\end{array}$ & $\mathrm{R}$ & 10.2 & $10-12,2-9$ & $\mathrm{~J}, \mathrm{G}, \mathrm{N}, \mathrm{C}$ \\
\hline 92 & Tree Flitter & $\begin{array}{l}\text { Hyarotis adarastus } \\
\text { Moore }\end{array}$ & VR & 3.4 & $11,2,4-9$ & $\mathrm{~J}, \mathrm{G}, \mathrm{N}$ \\
\hline 93 & Dark Palm Dart & Telicota ancilla Moore & $\mathrm{R}$ & 16.9 & $10-9$ & $\mathrm{~J}, \mathrm{G}, \mathrm{N}, \mathrm{C}$ \\
\hline 94 & Indian Palm Bob & Sauastrus gremius Fab. & VR & 4.4 & $10-11,5-9$ & $\mathrm{~J}, \mathrm{~N}, \mathrm{C}$ \\
\hline 95 & Giant Red Eye & Gangara thyrsis Fab. & VR & 0.1 & 8 & $\mathrm{~J}$ \\
\hline 96 & Smaller Dartlet & Oriens goloides Moore & VR & 0.5 & $7-8$ & $\mathrm{~J}$ \\
\hline 97 & Dusky Part Wing & Psolos fuligo Mabille & VR & 0.5 & $10,7-8$ & $\mathrm{~J}$ \\
\hline \multirow[t]{2}{*}{98} & $\begin{array}{l}\text { Obscure Branded } \\
\text { Swift }\end{array}$ & Pelopidas agna Moore & VR & 0.5 & 7 & $\mathrm{~J}$ \\
\hline & Acraeidae & & & & & \\
\hline \multirow[t]{2}{*}{99} & Tawny Coster & Acraia viole Fab. & $\mathrm{R}$ & 11.8 & $4-5$ & $\mathrm{~J}, \mathrm{G}, \mathrm{N}, \mathrm{C}$ \\
\hline & Amathusidae & & & & & \\
\hline 100 & Common Duffer & $\begin{array}{l}\text { Discophora sonaica } \\
\text { zal Westwood }\end{array}$ & VR & 0.1 & 11 & $\mathrm{~J}$ \\
\hline
\end{tabular}

$\mathrm{VC}=$ Very common, $\mathrm{C}=\mathrm{C}$ Common, $\mathrm{R}=\mathrm{Rare}, \mathrm{VR}=$ Very rare; $\mathrm{J}=\mathrm{JU}$ Campus, $\mathrm{G}=\mathrm{Gerua}, \mathrm{N}=$ Nabinagar,

$\mathrm{C}=$ Chandra; $*$ detailed in methods and materials

Table 2. Familywise diversity of butterfly species observed in four study areas

\begin{tabular}{l|c|c|c|c}
\hline Family & JU campus & Gerua & Nabinagar & Chandra \\
\hline Nymphalidae & 17 & 13 & 12 & 11 \\
Pieridae & 16 & 12 & 10 & 11 \\
Lycaenidae & 29 & 19 & 18 & 19 \\
Satyridae & 7 & 7 & 7 & 7 \\
Papilionidae & 7 & 7 & 7 & 7 \\
Danaidae & 5 & 4 & 4 & 5 \\
Hesperiidae & 15 & 8 & 10 & 7 \\
Acraeidae & 1 & 1 & 1 & 1 \\
Amathusidae & 1 & 0 & 0 & 0 \\
\hline Total & 98 & 71 & 69 & 68 \\
\hline
\end{tabular}


Table 3. Seasonal diversity of butterflies during 2012- 2013

\begin{tabular}{|c|c|c|c|c|c|c|c|c|c|c|c|c|}
\hline \multirow{4}{*}{$\begin{array}{l}\text { Butterfly } \\
\text { Family }\end{array}$} & \multicolumn{12}{|c|}{ Year and months } \\
\hline & \multicolumn{3}{|c|}{2012} & \multicolumn{9}{|c|}{2013} \\
\hline & Oct. & Nov & Dec. & Jan. & Feb. & Mar. & Apr. & May & Jun. & Jul. & Aug. & Sep. \\
\hline & \multicolumn{2}{|c|}{$\begin{array}{c}\text { Early } \\
\text { winter }\end{array}$} & \multicolumn{2}{|c|}{$\begin{array}{c}\text { Late } \\
\text { winter }\end{array}$} & \multicolumn{2}{|c|}{ Spring } & \multicolumn{2}{|c|}{ Summer } & \multicolumn{2}{|c|}{$\begin{array}{c}\text { Early } \\
\text { monsoon }\end{array}$} & \multicolumn{2}{|c|}{$\begin{array}{c}\text { Late } \\
\text { monsoon }\end{array}$} \\
\hline Nymphalidae & 10 & 9 & 8 & 5 & 14 & 14 & 15 & 15 & 10 & 11 & 12 & 9 \\
\hline Pieridae & 8 & 10 & 10 & 4 & 12 & 15 & 15 & 11 & 10 & 8 & 8 & 9 \\
\hline Lycaenidae & 20 & 19 & 18 & 10 & 22 & 20 & 20 & 21 & 23 & 19 & 20 & 18 \\
\hline Satyridae & 6 & 7 & 7 & 7 & 7 & 7 & 6 & 7 & 7 & 6 & 6 & 6 \\
\hline Papilionidae & 6 & 7 & 7 & 4 & 6 & 7 & 7 & 7 & 7 & 6 & 6 & 6 \\
\hline Danaidae & 3 & 4 & 4 & 1 & 2 & 4 & 4 & 4 & 4 & 4 & 4 & 3 \\
\hline Hesperiidae & 7 & 8 & 5 & 1 & 10 & 9 & 8 & 8 & 10 & 12 & 12 & 7 \\
\hline Acraeidae & 0 & 0 & 0 & 0 & 0 & 0 & 1 & 1 & 0 & 0 & 0 & 0 \\
\hline Amathusidae & 0 & 1 & 0 & 0 & 0 & 0 & 0 & 0 & 0 & 0 & 0 & 0 \\
\hline Total & 60 & 65 & 59 & 32 & 73 & 76 & 76 & 74 & 71 & 66 & 68 & 58 \\
\hline
\end{tabular}

Previously, an investigation from JU campus reported 51 species, where 21 species were identified as new records from Bangladesh (Hossain et al., 2003). Among 51 species, 39 were similar compared with the present study. Besides, Razzak et al. (2004) reported 80 species of butterfly belonging to 10 families from JU Campus of which 36 species were new records from Bangladesh. Among 80 species reported by Razzak, 64 species were similar to the present finding. Shefa \& Hossain (2010) reported 4 more new species from Bangladesh in a survey from JU Campus of which Taracus nara, Junonia orithya and Zizula hylax were similar to the present finding (Table 1). On the other hand, a total of 89 species under 10 families were recorded from JU Campus by Asaduzzaman (2011) of which 70 species were similar to the present findings (Table 1).

Islam et al. (2011) conducted an investigation in four different areas of Savar as, Krishnopur, AERE (Atomic Energy Research Establishment), Horters (part of JU Campus) and Rajalak Farm those were not overlapped with the selected areas of present investigation. They identified 158 species of butterfly under 10 families. Out of 158 species, 74 species were similar to the present findings (Table 1). They showed Delias hyparete, Mycaelesis mineus, Acraia viole and Neptis jumbah as very common (VC) species where these species were very rare (VR) compared to present findings (Table: 1). On the other hand, Islam et al. (2011) showed Neopithecops zalmora, Catopsilia pomona, Mycaelesis perseus as rare (R), but in present finding these species were common (C) (Table 1). Present record of 100 butterfly species in JU campus and its suburbs indicates that the diversity of butterfly has been increasing to greater extent. The JU campus is rich of its own diversity of vegetation types (Hossain et al., 1995). This diverse ecological habitat includes woodlands, climbers, vines, grasses, shrubs and herbs. The reason for increase of butterfly diversity in JU Campus might be due to its vegetation type (larval host plants and other vegetations for nectar and shelter of butterflies), soil condition, lake and favorable climate conditions (Hossain et al., 2003; Bashar et al., 2003; Razzak, et al., 2007; Tiple, 2009; Shefa \& Hossain, 2010; Chowdhury \& Hossain, 2013). In this present 
study, lowest number of butterfly species was recorded at Chandra (68 species) and highest number was recorded at JU Campus (98 species) (Table 2). Nabinagar and Chandra were more vulnerable to butterflies, because of the introduction of exotic plant species and monocultures (Nagy et al., 1998; Kocher, 2000; Marini-Filho et al., 2010; Majumder et al., 2013), contamination of soil, air and water quality by disposal of industrial waste and chemicals (Rima, 2014), human interference (Kocher \& Williams, 2000).

Natural habitat selection of butterflies is directly related to the availability of preferred food plants for larvae and adults (Grossmueller \& Lederhouse, 1987; Thomas, 1995). But it was observed in the present study that the natural plant habitat being converted with exotic plant species (Acacia sp) in Chandra (Sal forest) and monocultures that may be responsible for the poor species richness and diversity of butterfly (Nagy et al., 1998; Kocher, 2000; Marini-Filho et al., 2010; Majumder et al., 2013). Acacia sp. was the most dominant plants at Chandra (Sal Forest) and this exotic species was also observed at minimum level in JU Campus. Acacia sp. is threatening to native habitats by competing with indigenous vegetation and it is also responsible for the alteration of microbial soil functionalities and the early development of a native tree species (Boudiaf et. al., 2013). Although Acacia plant has many more bad effects to environment but in the present investigation it was observed that Acacia auriculiformis (Akashmoni) also act as larval host plant of a butterfly, Common Acacia blue (Family: Lycaenidae) (Monwar Hossain personal communication).

The changes in the diversity of butterflies occur due to various causes. Short-term changes may occur due to variation in weather, whereas long term changes due to modification of habitat quality and availability of larval host plants (Kocher et al., 2000). Butterflies are highly season specific. During the adverse condition of weather, in late winter (December, 2012-January, 2013) low population of butterfly was maintained due to scarcity of water, drying of grasses and other nectar feeding plants, continuing cold weather and low moisture (Table 3 ). The optimum time for butterfly species richness was from March, 2013 to June, 2013) (Table 3). The reason for increase of diversity might be the availability of nectarine plants such as Ixora chinensis, Lantana camara, Curcuma sp., Ageratum conyzoides, Mykania sp and so on. Those are good food sources of adult butterflies in early winter and the availability of larval host plants like Citrus sp, Madhuca indica, Mimusops elengi, Neolamarckia cadamba, Polyalthia longifolia, Mangifera indica, Artocarpus heterophyllus etc. in spring and summer as well as favorable weather conditions (Farjana Akter - personal observation). The availability of adult and larval food plants is the main biotic factor to retain butterfly diversity (Ruszczyk, 1986b).

The riches diversity of butterfly species indicates the good and healthy environment since butterfly acts as biotic indicators to any environmental change and habitat structure changes (Brown \& Chippendable, 1974; Thomas, 2005; Gross et al., 2007). The presence of more herbaceous plants makes opportunity and support for most of the butterfly. Due to existing of various economic and ecological importance (good indicator of climatic change and pollinator), butterfly conservation is necessary. Therefore to save our flying 
beauty and to enrich the population of butterfly, awareness buildup among mass people is necessary along with concern authorities should take necessary measures at greater extent to maintain the sustainability of our ecosystem.

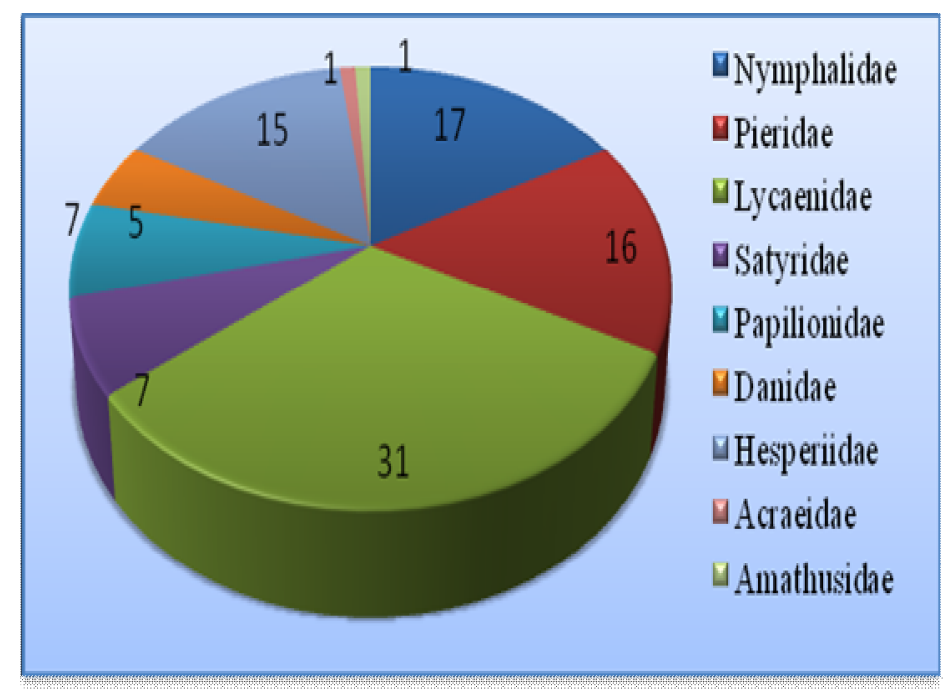

Fig. 2. Incidence (number) of butterflies among different families
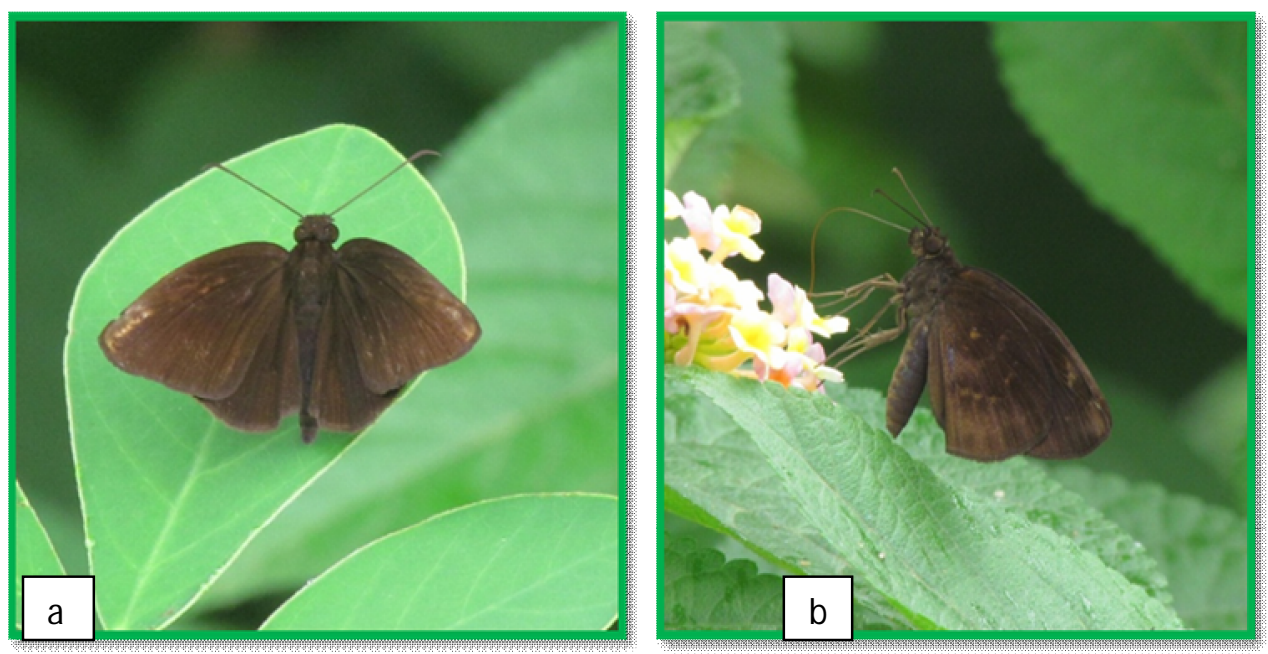

Plate 1. Dusky Part Wing (Psulos fuligo), (a) Dorsal side and (b) Ventral side

Acknowledgements: Thanks to Md. Abdur Rahim, technical officer, Department of Botany, Jahangirnagar University for providing support to identify various butterfly interacted plants. We are also grateful to the Department of Geography and Environment, Jahangirnagar University for giving data on weather condition. 


\section{REFERENCES}

Ahmad, M., Kabir, S.M.H., Ahmed, A.T.A., Rahman, A.K.A., Ahmed Z.U., Begum, Z.N.T., Hassan, M.A. and Khondker, M. (eds.). 2009. Encyclopedia of Flora and Fauna of Bangladesh, Vol. 21. Pterygota (Part). Asiatic Society of Bangladesh, Dhaka. 460 pp.

Alam, M.S. and Ullah, R.G.M. 1995. Butterflies of Chittagong University area - A check list. Bangladesh J Zool. 23 (1):111-112.

Ameen M and Chowdhury SH. 1968. A systematic account of insect fauna of Dacca city and its suburbs. 1. Papilionidae (Butterflies), Lepidoptera. J Asiatic Soc Pak; 13(2):221-227

Asaduzzaman, MD. 2011. Butterfly-plant interactions in the campus of Jahangirnagar University, Bangladesh (M. Sc Thesis, JU).

Baksha, M.W. and Chowdhury, J.H. 1983. Entomo-fauna in the forest of Bangladesh. 1. Pieridae: Lepidoptera. Univ J Zool Rajshahi Univ. 4:1-7.

Baksha, M.W. and Chowdhury, J.H. 1985. Entomo-fauna in the forest of Bangladesh. 11. Papilionidae: Lepidoptera. Univ J Zool Rajshahi Univ. 2:53-60.

Bashar, M.A., Mamun, M.A., Aslam, A.F.M. and Chowdhury, A.K. 2006. Biodiversity maintenance and conservation of butterfly- Plant association in some forests of Bangladesh. Bangladesh J. Zool. 34 (1) 55-67

Bashar, M.A. 2014. Butterflies of Bangladesh. Vol. I: Papilionidae, Nymphalidae, Pieridae, Danaidae and Lycaenidae. Biodiversity Conservation Trust Foundation publishing. University of Dhaka, Dhaka, Bangladesh. 1- 514 pp.

Boudiaf, I., Baudoin, E., Sanguin, H., Beddiar, A., Thioulouse, J., Galiana, A. , Prin, Y., Le Roux, C., Lebrun, M. and Duponnois, R. 2013. Soil Biology and Biochemistry. 65: $172-179$.

Bingham, C.T. 1905. Butterflies, Vol. I. Fauna of British India. Taylor and Francis, London. $511 \mathrm{pp}$.

Bingham, C.T. 1907. Butterflies, Vol. II. Fauna of British India. Taylor and Francis, London. 480pp.

Borges, R.M., V. Gowda, V. and M. Zacharias, M. 2003. Butterfly pollination and high contrast visual signals in a low-density distylous plant. Oecologia. 136: 571-573.

Brown, J.J. and Chippendale, G.M. 1974. Migration of the monarch butterfly, Danaus plexippus: Energy sources. Journal of Insect Physiology. 20(7): 1117-1130.

Chowdhury, S.H. and Hossain, M. 2011. Butterflies of Bangladesh: A Pictorial Handbook. Dhaka, Bangladesh. 1-201 pp.

Chowdhury, S.H. and Hossain, M. 2013. Butterflies of Bangladesh $\left(2^{\text {nd }}\right.$ ed. Revised and Enlarged version): A Pictorial Handbook. Dhaka, Bangladesh. 260 pp.

Chowdhury SH and Mohiuddin M. 2003. Butterflies of the eastern border of Bangladesh-a checklist. Rajshahi Univ J Zool. 22:1-9.

Evans, W.H. 1932. The Identification of Indian Butterflies. ( $2^{\text {nd }}$ ed. Revised). Published by- The Bombay Natural History Society. 454 pp.

Gross, K., Kalendra, E.J., Hudgens, B.R. and Haddad, N.M. 2007. Robustness and uncertainty in estimates of butterfly abundance from transect counts. Popul Ecol. 49:191-200.

Grossmueller, D.W. and Lederhouse, R.C. 1987. The role of nectar source distribution in habitat use and oviposition by the tiger swallowtail butterfly. Journal of Lepidopteran Society. 41(3):159-165.

Habib, M.S.A., Chowdhury, M.A., Islam, M.S., Mamun, S.H. and Iqbal, K.F. 2012. Butterflies of Bangladesh, Inventory- First Phase. Ecolife Initiatives. 63 pp.

Habib, M.S.A., Islam, M.S., Bhattacharjee, A., Jewel, M.M., Haque, S.N., Iqbal, K.F., Saif, S. and Chowdhury, M.A. 2013. Butterflies of Bangladesh, Inventory-Second Phase. Jiban Bikash Karjocrom. 95 pp. 
Hossain, M.M., Shaheduzzaman, M., Howlader. A.J. and Chowdhury, S.H. 2003. Check List of Butterflies of Jahangirnagar University, Bangladesh. Bangladesh Journal of life Science. 15(1): 83-86.

Hossain, M. 2014a. Check list of butterflies of the Sundarbans mangrove forest, Bangladesh. Journal of Entomology and Zoology Studies. 2(1):29-32.

Hossain, M. 2014b. Rediscovery of a butterfly, Neptis soma shania Evans 1924 (Lepidoptera: Nymphalidae) in the Sundarbans of Bangladesh. International Journal of Fauna and Biological Studies. 1 (3): 08-10.

Houlahan, J.E., Findlay, C.S., Schidt, B.R., Meyer, A.H. and Kuzmin,S.L. (2000) Quantitative evidence for global amphibian population declines. Nature. 404: 752-755.

Islam, A.T.M.F., Islam, M.H., Saifullah, A.S. M., Endo, K., and Akira, Y. 2011. New records of butterflies and their species diversity in four different areas of Savar, Univ. J. Zool. Rajshahi Univ. 30: 09-15.

Islam, M. A., Parven, N., Islam M.S. and Bashar, M.A. 2013. Butterfly abundance in relation to abiotic-biotic factors of forest ecosystem of the butterfly research park, Gazipur, Bangladesh. Bangladesh J Zool. 41(2): 247-255.

Khandokar, F., Rashid, M., Das, D.K. and Hossain, M. 2013. Species diversity and abundance of Butterflies in the Lawachara National Park, Bangladesh. Jahangirnagar University J. Biol. Sci. 2(2): 121-127.

Kocher, S.D. and Williams, E.H. 2000. The diversity and abundance of North American butterflies vary with habitat disturbance and geography. USA Journal of Biogeography. 27:785-794.

Kunte, K.J. 1997. Seasonal patterns in butterfly abundance and species diversity in four Tropical habitats in northern western Ghats. Journal of Biological Sciences. 22(5): 593-603.

Larsen, T.B. 2004. Butterflies of Bangladesh- an annotated checklist. IUCN Bangladesh. 158 $\mathrm{pp}$

Mac Nally, R., Fleishman, E., Bulluck, L.P. and Betrus, C.J. 2004. Comparative influence of spatial scale on beta diversity within regional assemblages of birds and butterflies. $J$. Biogeogr. 31, 917-929.

Majumder, J., Lodh, R. and Agarwala, B. K. 2013. Butterfly Species Richness and Diversity in the Trishna Wildlife Sanctuary in South Asia. The Journal of Insect Science. 13: 79.

Marini-Filho, O.J. and Martins, R.P. 2010. Nymphalid butte dispersal among forest fragments at Serra da Canastra National Park, Brazil. Journal of Insect Conservation. 14(4): 401-411.

Nagy, K.A., Henen, B.T. and Vyas, D.B. 1998. Nutritional quality of native and introduced food plants of wild desert tortoises. Journal of Herpetology. 32: 260-267.

Neogi, A.K., Baki, M.A. , Sadat, M.N., Selim, S. R. and Bhouiyan, N.A. 2014. Five New Records of Butterfly Species from Dhaka, Pirojpur and Cox'S Bazar Districts in Bangladesh. Journal of Entomology and Zoology Studies. 2 (3):197-200.

Rajagopal, T., Sekar, M., Manimozhi, A., Baskar, N. and Archunan, G. 2011. Diversity and community structure of butterfly of Arignar Anna Zoological Park, Chennai, Tamil Nadu. J. Environ. Biol. 201-207.

Razzak, M.A., Islam, A.T.M.F., Saifullah, A.S.M., Hossain, M.M., Shahjahan, R.M., Akira Yamanaka, A., and Endo, K.. 2007. A list of butterfly fauna in Jahangirnagar University Campus in Bangladesh. Nuclear Science and Applications. 16(2): 99-105.

Rima, N. 2014. Physiochemical analysis of puddling ground of butterflies in the campus of Jahangirnagar University (M.Sc Thesis, JU).

Robbins, R.K. and Opler, P.A. 1997. Butterfly diversity and a preliminary comparison with bird and mammal diversity. In: Biodiversity II, understanding and protecting our biological resources,

Wilson, D.E., M.L. Reaka-Kudla and E.O.Wilson (Eds.). Joseph Henry Press, Washington, DC. 
Ruszczyk, A. 1986b. Distribution and abundance of butterflies in the urbanization zones of porto Alegre, Brazil. Journal of the Research on the Lepidoptera. 25(3): 157-178.

Shefa, K. and Hossain, M.M. 2010. New records of butterflies from the Jahangirnagar University campus in Bangladesh. Bangladesh J. of Life Science. 22: 20-27.

Stuart, S.N., Chanson, J.S., Cox, N.A., Young, B.E., Rodrigues, A.S.L., Fischman, D.L. and Waller, R.W. (2004) Status and trends of amphibian declines and extinctions worldwide. Science. 306, 1783-1786 pp.

Talbot, G. 1978a. The Fauna of British India including Ceylone and Burma. Butterflies: Vol. I. Today and Tomorrow's Printers \& Publishers, New Delhi, India ( $2^{\text {nd }}$ reprint). 660 pp.

Talbot, G. 1978b. The Fauna of British India including Ceylone and Burma. Butterflies: Vol. I. Today and Tomorrow's Printers \& Publishers, New Delhi, India ( $2^{\text {nd }}$ reprint). 506 pp.

Thomas, J.A. 1995. The ecology and conservation of Maculinea arion and other European speciesof large blue butterfly. In: Pullin AS, editor. Ecology and Conservation of Butterflies. Chapman and Hall. 180-210 pp.

Thomas, J.A. 2005. Monitoring change in the abundance and distribution of insects using butterflies and other indicator groups. Philos Trans $R$ Soc B. 360:357.

Tiple, A.D. and Khurad, A.M. 2009. Butterfly Species Diversity, Habitats and Seasonal Distribution in and around Nagpur City, Central India. World J of Zool. 4(3): 153-162.

Wynter-Blyth, M.A. 1957. Butterflies of the Indian Region. Bombay Natural History Society, Bombay. 523pp. 